\title{
Sexual Victimization, Fear of Sexual Powerlessness, and Cognitive Emotion Dysregulation as Barriers to Sexual Assertiveness in College Women
}

Violence Against Women $201 X$, Vol XX(X) I-20

(C) The Author(s) 2013

Reprints and permissions: sagepub.com/journalsPermissions.nav DOI: $10.1177 / 1077801213517566$ vaw.sagepub.com

(SAGE

\section{Noga Zerubavel ${ }^{1,2}$ and Terri L. Messman-Moore ${ }^{2}$}

\begin{abstract}
The current study examined sexual victimization and two barriers to young women's sexual assertiveness: fear of sexual powerlessness and cognitive emotion dysregulation. College women $(N=499)$ responded to surveys and indicated that fear of sexual powerlessness and, to a lesser extent, cognitive emotion dysregulation were barriers to sexual assertiveness. Compared with nonvictims, sexually victimized women had greater problems with sexual assertiveness, fear of sexual powerlessness, and cognitive emotion dysregulation. Among victims, fear of sexual powerlessness and emotion dysregulation interacted to impede sexual assertiveness. Findings support targeting identified barriers in interventions to improve sexual assertiveness and reduce risk for unwanted sexual experiences and sexual victimization.
\end{abstract}

\section{Keywords}

cognitive emotion dysregulation, sexual assertiveness, sexual powerlessness, sexual victimization

Approximately $15 \%$ to $25 \%$ of college women are sexually victimized (Fisher, Cullen, \& Turner, 2000; Krebs, Lindquist, Warner, Fisher, \& Martin, 2007). Sexual victimization most often occurs not in a dark alley, but in a dorm room or apartment - contexts in which women primarily focus on a social relationship. Indeed, over $90 \%$ of college

'Duke University Medical Center, Durham, NC, USA

2Miami University, Oxford, OH, USA

Corresponding Author:

Noga Zerubavel, Department of Psychiatry and Behavioral Sciences, Duke University Medical Center, Box 3026, 2213 Elba Street, Durham, NC, 277I0, USA.

Email: noga.zerubavel@duke.edu 
rape victims know their assailant (Fisher et al., 2000; Ullman, Karabatsos, \& Koss, 1999). Thus, sexual victimization occurs within normal, familiar situations in the contexts of socializing and dating (Nurius, 2000), and it is often social and relational factors that prevent women from using self-protective behavior to deter sexual victimization (Rozee \& Koss, 2001). Yet, these are the very situations in which sexual assertiveness may help a young woman avoid sexual victimization. While a sexually assertive response may not always eliminate potential danger, verbal and physical sexual assertiveness can reduce the threat or extent of sexual victimization (Rozee \& Koss, 2001; Ullman, 1998), particularly with known assailants (Krebs et al., 2007). Nonetheless, many young women struggle to engage in sexually assertive behavior (Nurius, 2000). The purpose of the present study is to identify factors that may inhibit young women's sexual assertiveness, with a focus on two potential barriers: fear of sexual powerlessness and cognitive aspects of emotion dysregulation (e.g., emotional nonacceptance, lack of emotional awareness).

\section{Sexual Assertiveness}

We conceptualize sexual assertiveness as being able to recognize, prioritize, and express one's own limits, needs, and desires in a sexual situation. For young women, sexual situations are often tinged with confusion as they negotiate conflicting messages about their ability and right to be sexually assertive. Feminist theory has pointed to the existence of traditional sexual scripts, embedded in American culture, which portray men as dominant and women as passive (Chung, 2007; Phillips, 2000; Reinholtz, Muehlenhard, Phelps, \& Satterfield, 1995). As such, women's sexual assertiveness is contradictory to traditional sexual scripts (Impett \& Peplau, 2003) and may be viewed as undermining heterosexual intimacy (Rudman \& Fairchild, 2007), perceptions that cultivate an atmosphere that promotes women's sexual compliance. Feminist researchers have shown that young women often preserve the peace in relationships through suppression of their needs and aspirations (Burton, Halpern-Felsher, Rankin, Rehm, \& Humphreys, 2011; Impett, Schooler, \& Tolman, 2006; Phillips, 2000). Investment models have shown that when women are deeply invested in preserving relationships, they are more apt to tolerate abusive behaviors in the relationship (Rhatigan \& Street, 2005). Indeed, compliance with unwanted sex is often related to investment in maintaining the relationship (Impett \& Peplau, 2003).

Education regarding sexual victimization risk reduction does not necessarily translate into utilizing such skills (Nurius, 2000). Additional research is needed to understand why women who report feeling knowledgeable about sexual assertiveness still may not respond assertively in unwanted sexual situations. For young women, navigating conflicting cultural imperatives may be confusing, particularly with regard to limiting sexual activity. Tolman (2002) interviewed adolescent girls about their first sexual experiences and noted a common theme of "it just happened," indicating a lack of sexual agency and sexual assertiveness (Impett et al., 2006). Because identity is still developing in emerging adulthood, gender normative behaviors are an important reference point for determining appropriate responses in sexual situations, creating gender 
role conflict for young women regarding the use of sexually assertive behavior (e.g., resistance, refusal; Burton et al., 2011).

To promote young women's safety and empowerment in sexual interactions, it is important to identify barriers to sexual assertiveness. Sexual assertiveness is multifaceted and is likely influenced by numerous factors promoting sexual compliance. Young women's great investment in cultivating and maintaining intimate relationships (Burton et al., 2011; Chung, 2007) is often a central barrier to assertive responding (Austin, 2005; Impett \& Peplau, 2003; Rhatigan \& Street, 2005). In addition, it is important to examine traditional aspects of sexual assertiveness, including a sense of sexual agency and confidence in communicating one's sexual limits and desires. One factor that may likely inhibit a sexually assertive response is the fear that one is powerless. It is to this focus that we turn next.

\section{Fear of Sexual Powerlessness}

Young women have heard repeated warnings regarding sexual activity from parents, peers, and the media. Sex is "usually perceived as being potentially or actually violent, exploitative and degrading" (Schmidt, 1995, p. 9), and many of the messages play on the stimulation of fear, linking sex and danger, often in hopes of limiting sexual behavior (Cahill, 2001; Phillips, 2000). Years of warnings about situations that "get out of hand," sexually transmitted infections, unwanted pregnancies, and men who will leave after they "get what they want" can foster a perception of sexual powerlessness in young women (Cahill, 2001; Phillips, 2000). Dominant cultural discourses such as the "pleasing woman" discourse, in which the woman is portrayed as compliant and selfsacrificing (Phillips, 2000), and the "male sexual drive" discourse, in which a man's sex drive is portrayed as uncontrollable once he is sufficiently aroused (Phillips, 2000; Reinholtz et al., 1995), reflect dominant cultural norms while sculpting women's views of sex and sexuality in ways that promote fear of sexual powerlessness. Through such messages, some women internalize fear of sexual powerlessness.

One fundamental basis for sexual assertiveness is having the belief that one can control a sexual situation. Individuals respond to conflict based not only on objective power, but also on the perception of power (Coleman, 2006). While research on women's sexual assertiveness has emphasized issues of gendered power asymmetry and perceptions of powerlessness, the affective response to those perceptions (i.e., fear) has not yet been explored. Yet fear associated with such perceptions may be critical to understanding women's sexual assertiveness. Fear of sexual powerlessness involves a woman's belief that she may not be able to control a sexual situation (i.e., lack of sexual self-efficacy). Research indicates that perceptions of sexual control and sexual assertiveness are interrelated, and doubts about sexual self-efficacy "serve as psychological barriers to assertively resisting sexual aggression" (Macy, Nurius, \& Norris, 2007, p. 545). Resistance efforts in dangerous sexual situations depend on a woman's appraisal of her own ability to control the situation (Nurius, 2000).

Although not all women experience fear of sexual powerlessness, it should be acknowledged that young women navigate a cultural context in which sex is often 
referenced with, and thus framed by, warnings regarding potential physical and relational risks. Research on fear appeals (scare tactics) consistently demonstrates that when people believe that they are incapable of successfully avoiding the threat and appraise the threat level as high (i.e., due to scare tactics), they allocate resources to control their fear rather than to avoid the potential danger, thereby inhibiting safety behavior (Cho \& Salmon, 2006; Witte \& Morrison, 1995). Given that fear of sexual powerlessness involves a perception of high threat and low self-efficacy, we predict that women with greater fear of sexual powerlessness will allocate their psychic resources toward emotion regulation (in an effort to reduce fear) rather than addressing potential danger via self-protective (i.e., sexually assertive) responses. Whether that allocation is problematic may depend on how well emotion is regulated.

\section{Cognitive Emotion Dysregulation}

Difficulties with emotion regulation may play a critical role in the link between fear of sexual powerlessness and sexual assertiveness. The sense of agency that is often generated in the midst of powerful emotions (Austin, 2005) may be absent without awareness or understanding of surges of emotion, or negated by rejection of emotional experience. When one ignores or rejects emotional experience, information-providing functions of emotions are not carried out intra- or interpersonally, increasing risk for sexual miscommunication or worse, sexual victimization. Furthermore, given traditional sexual scripts that outline socially prescribed suitable behavior in sexual situations (O'Sullivan \& Byers, 1996), defining women's gender role as passive and nonassertive (Impett \& Peplau, 2003; Phillips, 2000), emotional experience would tend to be modulated in a socially normative manner, making sexual assertiveness less likely.

Emotion dysregulation includes several components, such as difficulty modifying, modulating, and tolerating emotional experiences (Gratz \& Roemer, 2004; Linehan, 1993). Gratz and Roemer's (2004) integrated model of emotion dysregulation highlights clinically informative components of behavioral and cognitive emotion dysregulation, providing a framework that is useful in explaining both intra- and interpersonal difficulties. The cognitive aspects of emotion dysregulation within this model (Gratz \& Roemer, 2004) include a lack of emotional awareness, lack of emotional clarity, and nonacceptance of emotional responses. Cognitive emotion regulation has been emphasized as an important, clinically informative construct (Aldao \& Nolen-Hoeksema, 2010; Garnefski, Kraaij, \& Spinhoven, 2001). When cognitive emotion regulation strategies are accessible and effective (i.e., emotional awareness, clarity, and acceptance), an individual then has a mindful stance toward emotions (Linehan, 1993).

When sexual situations trigger a fear of sexual powerlessness, women must call on their ability to mindfully regulate emotion to manage that fear. Awareness of the emotion is necessary to initiate action, clarity guides response choice, and nonjudgmental acceptance of the emotion is requisite to generate an assertive response. Thus, for women with greater fear of sexual powerlessness, cognitive emotion dysregulation may be particularly detrimental. Awareness, understanding, and acceptance of emotional experience should facilitate sexual assertiveness, whereas cognitive emotion 
dysregulation would inhibit it. Without acknowledging and understanding an emotion, it cannot be regulated in an intentional manner (Stegge \& Terwogt, 2007), and the ability to respond to it is diminished (Isaacs, 1998). When individuals lack emotional awareness, they are likely to experience hesitation and uncertainty and may make lessaccurate assessments about situations (Isaacs, 1998). This could make the perception of risk, as well as the initiation of assertive behavior, both difficult and less likely. Furthermore, if a woman does not accept her emotional experience, she is more likely to try to distract herself or to quash the emotion, ineffectively allocating psychic resources (Tice \& Bratslavsky, 2000). In this way, nonacceptance can be conceptualized as accommodating and promoting emotional avoidance (Blackledge \& Hayes, 2001) and would be likely to debilitate sexual assertiveness. As such, a woman who feels fear in a sexual situation but does not accept her emotions may allocate her psychic resources elsewhere, in effect directing them away from thinking about possible self-protective options.

\section{Women With a History of Sexual Victimization}

Understanding barriers to sexual assertiveness is particularly relevant for women with a history of sexual victimization (referred to as victims for brevity). Longitudinal studies have consistently demonstrated a reciprocal relationship between these factors; history of sexual victimization leads to diminished sexual assertiveness, and difficulty with sexual assertiveness predicts later sexual victimization (Greene \& Navarro, 1998; Livingston, Testa, \& VanZile-Tamsen, 2007). Furthermore, among victims, sex often becomes associated with fear (Foa \& Rothbaum, 1998). Foa, Zinbarg, and Rothbaum (1992) theorized that after suffering powerlessness in the face of a traumatic event, one is more likely to expect to be helpless in the future. Herman (1992) noted that helplessness during trauma often leads to heightened sensitivity toward feelings of helplessness and fear of sexual powerlessness. Moreover, past experience with a coercive male partner promotes the likelihood that, in future sexual situations, a woman will behave in a compliant manner to avoid conflict (Impett \& Peplau, 2003). An important distinguishing factor among women who have experienced sexual victimization is level of perceived powerlessness (Macy et al., 2007). Given this, it is important to determine whether victims are particularly vulnerable to difficulties with fear of sexual powerlessness and cognitive emotion dysregulation, thereby contributing to difficulties with sexual assertiveness.

\section{Hypotheses}

The study included a series of hypotheses addressing related research questions. The first hypothesis was that fear of sexual powerlessness acts as a barrier to sexual assertiveness. It was anticipated that women with high levels of fear of sexual powerlessness would have lower levels of sexual assertiveness, whereas those low in fear of sexual powerlessness would indicate higher levels of sexual assertiveness. The second hypothesis was that cognitive emotion dysregulation would contribute to difficulties 
with sexual assertiveness. Women with higher levels of cognitive emotion dysregulation should have lower levels of sexual assertiveness, while those with greater mindful emotion regulation (less cognitive emotion dysregulation) would have higher levels of sexual assertiveness. Because mindful emotion regulation may facilitate a sexually assertive response in the context of fear of sexual powerlessness, it was hypothesized that cognitive emotion dysregulation would moderate the relationship between fear of sexual powerlessness and sexual assertiveness, such that the fear may either promote or inhibit sexual assertiveness, depending on levels of emotion regulation. It was anticipated that in the context of cognitive emotion dysregulation, fear of sexual powerlessness would have a debilitating impact on sexual assertiveness, whereas in the context of mindful emotion regulation (low cognitive emotion dysregulation), fear may function in a protective manner, mobilizing an assertive response.

It is critical to consider the relationship between history of sexual victimization and the factors examined here. It was hypothesized that victims would have higher levels of fear of sexual powerlessness, greater cognitive emotion dysregulation, and lower levels of sexual assertiveness than nonvictims. As discussed earlier, sexual victimization is often associated with decreased sexual assertiveness (e.g., Livingston et al., 2007). However, less is known about whether the patterns hypothesized regarding fear of sexual powerlessness and cognitive emotion dysregulation would be the same for women with and without a history of sexual victimization. Thus, it seems important to examine the extent to which associations among variables differ for these two groups.

\section{Method}

\section{Participants}

Participants were 499 undergraduate women between the ages of 18 and $23(M=$ $18.73, S D=0.71)$ at a medium-sized, Midwestern university. The majority of participants $(63.7 \%)$ were freshmen, $93.8 \%$ were Caucasian, $70.1 \%$ were Catholic or Protestant, and the majority reported upper middle class socioeconomic status.

\section{Procedure}

The university's Committee on Human Subjects in Research (CUHSR) authorized the procedures for this study. Women were recruited through an online system and received course credit in an introductory psychology class for participating. Participants gave informed consent and were debriefed following their participation. Data were collected in groups overseen by two female research assistants. Participant responses were anonymous.

\section{Measures}

Sexual assertiveness. Sexual assertiveness included three components: relational sexual assertiveness, sexual agency, and sexual compliance. The Sexual Assertiveness 
Questionnaire (SAQ; Messman-Moore, Ward, \& Walker, 2007; Walker, MessmanMoore, \& Ward, 2011) was used to assess the first two components. This self-report instrument has demonstrated good to excellent internal consistency as well as convergent and divergent validity. Participants used a 5-point Likert scale to indicate level of agreement with each statement. Sums of scale items were computed with higher scores signifying more adaptive functioning (i.e., greater sexual assertiveness).

The first component of sexual assertiveness, relational sexual assertiveness, was assessed with the Relational Sexual Assertiveness subscale (Cronbach's alpha $=.94$ ) of the SAQ. Items on this scale indicate a level of partner focus in sexual decision making, with agreement on reverse-scored statements such as: "I engage in unwanted sexual activity to avoid hurting my partner's feelings," and "I agree to have sex when I don't feel like it."

The second component of sexual assertiveness, sexual agency, was assessed using the Sexual Agency and Communication subscale (Cronbach's alpha $=.90$ ) of the SAQ, which indicates level of ability or comfort with sexual communication. High scores on sexual agency indicate agreement with statements such as "I know what I want sexually" and "I am good at expressing my sexual needs and wants."

The third component of sexual assertiveness, sexual compliance, was assessed with the Partner Approval subscale (Cronbach's alpha $=.91$ ) of the Sexual Motivation Scale (Cooper, Shapiro, \& Powers, 1998). Participants use a 5-point Likert scale to indicate how often they engage in sexual activity due to each motive. High scores of sexual compliance correspond with higher frequency reported on statements such as "How often do you have sex because you don't want your partner to be angry with you?" or "How often do you have sex because you're afraid that your partner will leave you if you don't?"

Fear of sexual powerlessness. Fear of sexual powerlessness was assessed using the control subscale of the Sexual Self-Esteem Inventory for Women Short Form (SSEI-W-S; Zeanah \& Schwarz, 1996). Participants are asked to rate their reactions to and feelings about their sexual experiences by indicating the degree of agreement with each statement on a 6-point Likert scale. The control subscale includes statements such as "I feel physically vulnerable in a sexual encounter" and "I worry that I won't be able to stop something I don't want to do in a sexual situation." The control subscale has good internal consistency (Zeanah \& Schwarz, 1996) and had a Cronbach's alpha of .84 in the current study.

Cognitive emotion dysregulation. Cognitive emotion dysregulation was assessed using the Difficulties in Emotion Regulation Scale (DERS; Gratz \& Roemer, 2004). The DERS is a 36-item self-report measure that has been shown to have good validity and reliability (Gratz \& Roemer, 2004). Participants use a 5-point Likert scale to indicate how often the statements apply to themselves. Sum scores are computed such that higher scores signify more extensive emotion dysregulation. The DERS assesses six factors (three behavioral, three cognitive) of emotion dysregulation, three of which were summed to create a cognitive emotion dysregulation score: (a) Lack 
of Emotional Awareness $(\alpha=.83)$, (b) Lack of Emotional Clarity $(\alpha=.82)$, and (c) Nonacceptance of Emotional Responses $(\alpha=.90)$. The Cognitive Emotion Dysregulation scale included 17 items (e.g., "I pay attention to how I feel" (reverse-scored), "I have difficulty making sense out of my feelings," and "When I am upset, I become irritated with myself for feeling that way") and demonstrated strong internal reliability $(\alpha=.89)$.

Adult sexual victimization. Adult sexual victimization was assessed with a modified version of the Sexual Experiences Survey (SES; Koss, Gidycz, \& Wisniewski, 1987). The SES asks questions regarding unwanted sexual contact, sexual coercion, and experiences of attempted and completed rape since the age of 14. The SES has demonstrated strong internal validity and test-retest reliability (Koss et al., 1987). The original 10-item SES was modified to include 14 additional questions assessing for various forms of sexual contact (e.g., kissing and fondling, oral-genital contact), attempted or accomplished through various methods of coercion (e.g., persistent arguments, physical force). Questions regarding alcohol and substance use were altered using phrasing developed by Muehlenhard, Powch, Phelps, and Giusti (1992).

Consistent with previous usage, sexual victimization was coded as a 2-level (yes/ no) variable. A participant was considered victimized if she reported being subjected to nonconsensual sexual activity that included oral sex, vaginal or anal intercourse due to verbal coercion, threats or use of physical force, or because she was incapable of giving consent or resisting due to alcohol or drugs. Only completed sexual victimization was included, as sexual assertiveness may play a role in the distinction between attempted and completed victimization (e.g., Fisher et al., 2000; Rozee \& Koss, 2001; Ullman, 1998).

\section{Results}

\section{Preliminary Analyses}

The original sample consisted of 546 women. After coding data regarding sexual victimization, women who could not be unambiguously classified as victims or nonvictims of sexual assault were eliminated from data analysis. Of the original sample, 44 women were excluded due to missing data on the sexual victimization measure or failure to report victimization to our criteria (e.g., reporting attempted but not completed experiences, or fondling, but not penetration). Three other women were excluded due to describing experiences of child sexual abuse in elaborating their experiences of adult sexual victimization. Of the remaining 499 women, 39\% $(n=194)$ had a history of sexual victimization.

Pearson correlations indicated that all variables were significantly associated in the hypothesized direction. Lower levels of sexual assertiveness (and higher levels of sexual compliance) were associated with higher levels of fear of sexual powerlessness and greater cognitive emotion dysregulation (see Table 1). 
Table I. Pearson Correlations among Sexual Assertiveness, Fear of Sexual Powerlessness, and Cognitive Emotion Dysregulation.

\begin{tabular}{|c|c|c|c|c|c|}
\hline \multirow[b]{2}{*}{ Measure } & \multicolumn{3}{|c|}{ Sexual assertiveness } & \multirow{2}{*}{$\begin{array}{l}\begin{array}{l}\text { Fear of sexual } \\
\text { powerlessness }\end{array} \\
4\end{array}$} & \multirow{2}{*}{$\begin{array}{c}\begin{array}{c}\text { Cognitive } \\
\text { emotional } \\
\text { dysregulation }\end{array} \\
5\end{array}$} \\
\hline & I & 2 & 3 & & \\
\hline $\begin{array}{l}\text { I. Relational sexual } \\
\text { assertiveness }\end{array}$ & I & $.52 * * *$ & $-.54 * * *$ & $-.62 * * *$ & $-.34 * * *$ \\
\hline 2. Sexual agency & & $\mathrm{I}$ & $-.28 * * *$ & $-.58 * * *$ & $-.43 * * *$ \\
\hline 3. Sexual compliance & & & I & .47 **** & $.30 * * *$ \\
\hline $\begin{array}{l}\text { 4. Fear of sexual } \\
\text { powerlessness }\end{array}$ & & & & $\mathrm{I}$ & $.40 * * *$ \\
\hline $\begin{array}{l}\text { 5. Cognitive emotional } \\
\text { dysregulation }\end{array}$ & & & & & I \\
\hline$M(N=499)$ & 57.90 & 25.62 & 5.08 & 17.62 & 36.76 \\
\hline$S D$ & 11.01 & 6.36 & 2.36 & 7.12 & 10.00 \\
\hline Range & $16-70$ & $9-35$ & $4-19$ & $7-40$ & $17-67$ \\
\hline
\end{tabular}

Note. $* * * p<.001$.

Table 2. Means and Standard Deviations of All Variables.

\begin{tabular}{|c|c|c|c|c|c|c|}
\hline & \multicolumn{2}{|c|}{ Victims } & \multicolumn{2}{|c|}{ Nonvictims } & \multirow[b]{2}{*}{$F$} & \multirow{2}{*}{$\begin{array}{c}\text { Partial eta } \\
\text { squared }\end{array}$} \\
\hline & $M$ & $S D$ & $M$ & $S D$ & & \\
\hline $\begin{array}{l}\text { Relational sexual } \\
\text { assertiveness }\end{array}$ & 52.46 & 12.21 & 61.24 & 8.73 & $74.58 * * *$ & .15 \\
\hline Sexual agency & 24.77 & 6.60 & 26.07 & 6.29 & $4.18^{*}$ & .01 \\
\hline Sexual compliance & 5.92 & 2.98 & 4.49 & 1.50 & $42.43 * * *$ & .09 \\
\hline $\begin{array}{l}\text { Fear of sexual } \\
\text { powerlessness }\end{array}$ & 19.74 & 7.77 & 16.15 & 6.48 & $26.5 I^{* * * *}$ & .06 \\
\hline $\begin{array}{l}\text { Cognitive emotional } \\
\text { dysregulation }\end{array}$ & 38.14 & 9.97 & 35.89 & 9.94 & $5.18 *$ & .01 \\
\hline
\end{tabular}

Note. $* p<.05 . * * p<.01 . * * * p<.001$.

\section{Differences Between Victims and Nonvictims}

The next set of analyses evaluated whether victims differed from nonvictims on study variables. Results of the MANOVA indicated the presence of significant differences in sexual assertiveness, fear of sexual powerlessness, and emotion dysregulation (Wilks' $\lambda=.82, F(5,419)=18.18, p<.001$; see Table 2 ). For all aspects of sexual assertiveness, victims experienced more difficulty than nonvictims (all $p \mathrm{~s}<.05$ ), as indicated by lower relational sexual assertiveness and sexual agency and higher 
sexual compliance. Victims also indicated greater fear of sexual powerlessness and more difficulty in cognitive emotion dysregulation than nonvictims. Effect sizes based on partial eta squared were in the large to medium range (Cohen, 1992) for relational sexual assertiveness (.15) and sexual compliance (.09).

\section{Interaction of Fear of Sexual Powerlessness and Cognitive Emotion Dysregulation}

Hierarchical regression analyses were used to determine whether cognitive emotion dysregulation moderates the relationship between fear of sexual powerlessness and sexual assertiveness. Analyses were conducted separately for victims and nonvictims, and three different sets of analyses were conducted for each aspect of sexual assertiveness. The moderation analyses were tested using continuous variables (Holmbeck, 1997), and potential issues of multi-collinearity were minimized by centering the independent variables (Frazier, Tix, \& Barron, 2004). For each analysis, the main effect and moderating variable were entered in the first step, and the interaction term was entered in the second step (Holmbeck, 1997). Significant moderation effects were determined by testing the statistical significance of the slopes for the simple regression lines for each significant interaction (Frazier et al., 2004; Holmbeck, 1997). As standardized $(\beta)$ regression coefficients for the interaction can not be interpreted (see Frazier et al., 2004), unstandardized (B) coefficients are also reported. Hierarchical regressions showed that overall (see Table 3), fear of sexual powerlessness and cognitive emotion dysregulation accounted for substantial amounts of variance in sexual assertiveness (Cohen, 1992), explaining more variance in sexual assertiveness among victims. Effect sizes were in the large range in victims, with corresponding small or medium effect sizes in nonvictims (Cohen, 1992).

Victims. Fear of sexual powerlessness predicted all aspects of sexual assertiveness, such that women with greater fear of sexual powerlessness endorsed greater sexual compliance and more difficulty with relational sexual assertiveness and sexual agency. Cognitive emotion dysregulation also predicted all aspects of sexual assertiveness, such that victims with more cognitive emotion dysregulation reported more sexual compliance and greater struggles with relational sexual assertiveness and sexual agency.

In addition, for victims, cognitive emotion dysregulation moderated the relationship between fear of sexual powerlessness and sexual compliance, $t(171)=3.34, p<$ .05 (see Figure 1). Victims with low fear of sexual powerlessness had lower levels of sexual compliance regardless of level of cognitive emotion dysregulation. In contrast, victims with high fear of sexual powerlessness had greater levels of sexual compliance, and the relationship was stronger in the context of greater cognitive emotion dysregulation. The interaction of fear of sexual powerlessness and cognitive emotion dysregulation accounted for an additional $5 \%$ of the variance in sexual compliance. Simple slope significance tests showed that at all levels of cognitive emotion 
Table 3. Examination of the Interaction of Fear of Sexual Powerlessness and Cognitive Emotion Dysregulation to Predict Sexual Assertiveness.

\begin{tabular}{|c|c|c|c|c|c|}
\hline \multicolumn{6}{|c|}{ Relational sexual assertiveness } \\
\hline \multicolumn{6}{|c|}{ Victims $(n=194)$} \\
\hline Step & $\beta$ & $B$ & $S E(B)$ & $F$ & $R^{2}$ \\
\hline I. Fear of sexual powerlessness & -.62 & $-.97 * * *$ & .10 & & \\
\hline Cognitive emotional dysregulation & -.13 & $-.16 *$ & .08 & 68.46 & .46 \\
\hline 2. FSP $\times$ CED & N/A & -.01 & .10 & 46.68 & .46 \\
\hline \multicolumn{6}{|c|}{ Nonvictims $(n=305)$} \\
\hline Step & $\beta$ & $B$ & $S E(B)$ & $F$ & $R^{2}$ \\
\hline I. Fear of sexual powerlessness & -.48 & $-.65 * * *$ & .08 & & \\
\hline Cognitive emotional dysregulation & -.09 & -.08 & .05 & 49.74 & .28 \\
\hline 2. FSP $\times$ CED & N/A & $<.01$ & .01 & 33.32 & .28 \\
\hline
\end{tabular}

\section{Sexual agency}

Victims $(n=194)$

\begin{tabular}{llllll}
\hline Step & $\beta$ & $B$ & $S E(B)$ & $F$ & $R^{2}$ \\
\hline I. Fear of sexual powerlessness & -.45 & $-.38^{* * * *}$ & .05 & & \\
Cognitive emotional dysregulation & -.35 & $-.23^{* * *}$ & .04 & 64.14 & .44 \\
2. FSP $\times$ CED & N/A & $<.01$ & .01 & 42.57 & .44 \\
\hline
\end{tabular}

Nonvictims $(n=305)$

\begin{tabular}{llllll}
\hline Step & $\beta$ & $B$ & $S E(B)$ & $F$ & $R^{2}$ \\
\hline I. Fear of sexual powerlessness & -.53 & $-.5 I^{* * *}$ & .05 & & \\
Cognitive emotional dysregulation & -.15 & $-.09 * *$ & .04 & 73.20 & .36 \\
2. FSP $\times$ CED & N/A & $<.01$ & .01 & 48.61 & .36 \\
\hline
\end{tabular}

\section{Sexual compliance}

Victims $(n=194)$

\begin{tabular}{llllll}
\hline Step & $\beta$ & $B$ & $S E(B)$ & $F$ & $R^{2}$ \\
\hline I. Fear of sexual powerlessness & -.47 & $.18^{* * * *}$ & .03 & & \\
Cognitive emotional dysregulation & -.23 & $.07^{* * * *}$ & .02 & 45.14 & .35 \\
2. FSP $\times$ CED & N/A & $.01^{* * *}$ & $<.01$ & 35.60 & .40 \\
\hline
\end{tabular}

Nonvictims $(n=305)$

\begin{tabular}{llllll}
\hline Step & $\beta$ & $B$ & $S E(B)$ & $F$ & $R^{2}$ \\
\hline I. Fear of sexual powerlessness & .29 & $.07^{* * * *}$ & .02 & & \\
Cognitive emotional dysregulation & .04 & .01 & .01 & 14.02 & .10 \\
2. FSP $\times$ CED & N/A & $<.01$ & $<.01$ & 9.37 & .10 \\
\hline
\end{tabular}

Note. FSP = fear of sexual powerlessness, CED = cognitive emotion dysregulation.

$* p<.05 . *_{p}<.01$. $* * * p<.001$. 


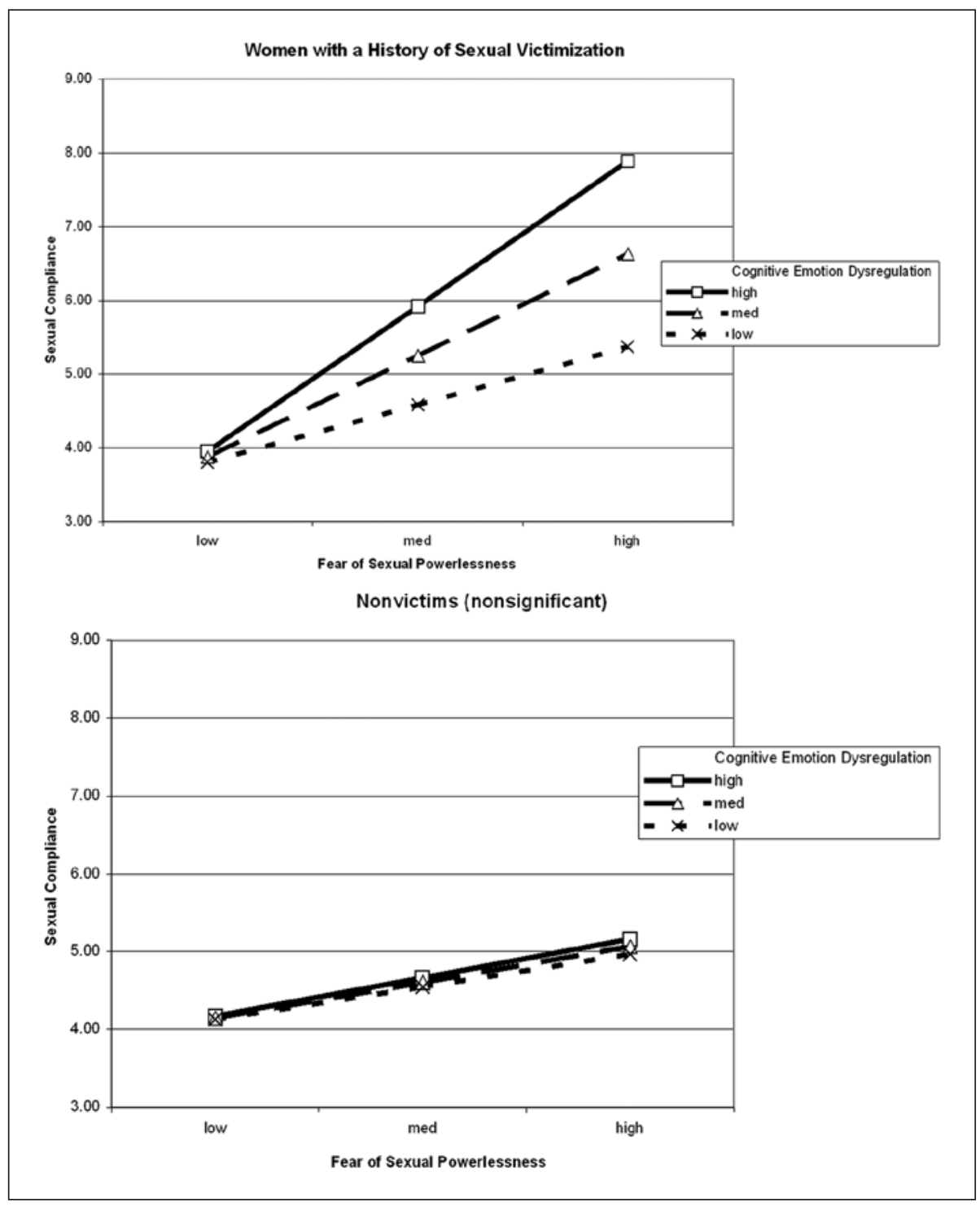

Figure I. The interaction of fear of sexual powerlessness and cognitive emotional dysregulation predicting sexual compliance.

dysregulation, as fear of sexual powerlessness increased, sexual compliance also rose $(p<.001)$. At low levels of fear of sexual powerlessness, cognitive emotion dysregulation was not associated with sexual compliance, whereas at high levels of fear of sexual powerlessness, greater cognitive emotion dysregulation increased the tendency 
toward sexual compliance. As moderation effects are extremely difficult to detect in nonexperimental studies and are usually unlikely to reach the required statistical significance (McClelland \& Judd, 1993), the significant interaction is noteworthy. Effect sizes for the interaction were in the large range for prediction of all sexual assertiveness variables $\left(R^{2}>.40\right.$; Cohen, 1992).

Nonvictims. Women with greater fear of sexual powerlessness endorsed greater sexual compliance and more problems with relational sexual assertiveness and sexual agency. Cognitive emotion dysregulation predicted sexual agency, but not relational sexual assertiveness or sexual compliance, and did not moderate the relationship between fear of sexual powerlessness and sexual assertiveness. Effect sizes were in the medium range for prediction of relational sexual assertiveness and sexual agency and communication $\left(R^{2}>.15\right.$; Cohen, 1992).

\section{Discussion}

For most young women, regardless of victimization status, fear of sexual powerlessness appears to be a major barrier to sexual assertiveness. In the midst of fears over whether one has any power in a sexual situation, it is extremely difficult for a young woman to express reservations, request that sexual activity be halted or altered, or leave an uncomfortable situation (Macy et al., 2007; Nurius, 2000). Indeed, fear of sexual powerlessness was a significant, strong predictor of every aspect of sexual assertiveness, individually and in concert with cognitive emotion dysregulation. In addition, cognitive emotion dysregulation also acts as a barrier to sexual assertiveness, particularly for victims. When resources are allocated to the regulation of internal experience (e.g., avoidance or negative evaluation of emotions), they are, in effect, allocated away from the management of the interpersonal experience (e.g., safety, boundary management). Indeed, for victims, fear of sexual powerlessness interacted with cognitive emotion dysregulation to increase levels of sexual compliance.

While not all women experience fear of sexual powerlessness, it is evident that for those who do, it negatively impacts sexual assertiveness. Fear of sexual powerlessness was associated with lower levels of relational sexual assertiveness and sexual agency, and greater sexual compliance. Furthermore, fear of sexual powerlessness appeared to impact all aspects of sexual assertiveness to a greater extent than did cognitive emotion dysregulation. These findings are consistent with earlier ideas that given a perceived lack of power, any conflict is considered within the frame of powerlessness (Coleman, 2006; Herman, 1992), and behavior that requires power (e.g., sexual assertiveness) is thereby likely to seem unrealistic or unattainable (Cahill, 2001; Impett \& Peplau, 2003; Reinholtz et al., 1995).

Counter to expectation, fear of sexual powerlessness, even in the context of mindful emotion regulation, did not motivate women to claim their sexual power; rather, for victims and nonvictims alike, such fears consistently undermined sexual assertiveness. This would suggest that the warnings of sexual powerlessness (Phillips, 2000; 
Reinholtz et al., 1995) — that is, scare tactics likely intended to encourage women to be cautious and avoid dangerous or risky sexual situations (Cahill, 2001; Kyes, 1995; Schmidt, 1995) — may not serve a protective function (e.g., to encourage sexual assertiveness). Results here are consistent with what we have learned from public health research on fear appeals (Cho \& Salmon, 2006; Witte \& Morrison, 1995), that within a context of high threat (e.g., social milieu of women's sexual powerlessness; Cahill, 2001; Phillips, 2000; Reinholtz et al., 1995), women with low self-efficacy (e.g., fear of sexual powerlessness) are particularly unlikely to behave in ways that aim to manage the threat (e.g., sexual assertiveness). Thus, an important message of this study is that we must change the cultural milieu of women's sexual powerlessness; specifically, we must help young women develop an improved sense of sexual self-efficacy without using scare tactics.

\section{The Impact of Sexual Victimization}

Comparative analyses indicated that women who had a history of sexual victimization, even if functioning quite highly (e.g., attending college), are nonetheless affected by the experience. Victims had greater fear of sexual powerlessness than nonvictims, and previous experiences of sexual powerlessness have likely contributed to the fear that such situations will arise again. Consistent with research on sexual victimization among college women with a history of childhood sexual abuse (Messman-Moore, Walsh, \& DiLillo, 2010), victims also had greater cognitive emotion dysregulation than nonvictims, suggesting that they were generally less emotionally aware, had less emotional clarity, and had a more critical, judgmental stance toward their emotions. It is also evident from these results that victims reported greater problems with all aspects of sexual assertiveness, consistent with earlier research (Greene \& Navarro, 1998; Livingston et al., 2007). Interpretation of effect sizes suggests that relational sexual assertiveness and sexual compliance were the predictors most strongly associated with history of sexual victimization. These aspects of sexual assertiveness are particularly critical for women, given that most sexual victimization takes place with known assailants and thus in the context of an existing social or intimate relationship (Krebs et al., 2007; Impett \& Peplau, 2003; Nurius, 2000).

In most cases, fear of sexual powerlessness and cognitive emotion dysregulation had independent effects on sexual assertiveness. Fear of sexual powerlessness predicted all aspects of sexual assertiveness for victims and nonvictims. However, cognitive emotion dysregulation predicted only sexual agency among nonvictims, although it had a more wide-reaching impact among victims. Among victims, results regarding fear of sexual powerlessness and cognitive emotion dysregulation suggest the two problems have a synergistic effect, enhancing the tendency toward sexual compliance. When victims had greater doubt regarding their own ability to control and limit sexual activity, and lacked emotional awareness/understanding and negatively evaluated their emotions, they reported a greater likelihood of engaging in unwanted sex to please a partner. The opposite was not true; findings indicated that 
mindful emotion regulation did not serve a protective function of enhancing selfprotective responses.

\section{Limitations and Future Directions}

The current study is one of the first to explicitly examine how emotion dysregulation may impede healthy, self-protective, and sexually assertive behavior in young women. Yet, despite its novelty, the interpretation of findings should be tempered by several limitations. The study focused on a cultural context specific to young, heterosexual, American college women. While this constraint may limit generalization of findings to women in other developmental stages, the age range may not be problematic given that women in this age group are most at risk for sexual victimization (Fisher et al., 2000; Krebs et al., 2007). Thus, this demographic represents a target population for continued study. However, fear of sexual powerlessness, emotion dysregulation, and assertiveness should also be examined among other groups, including lesbian and bisexual women, as well as women in other age ranges.

Future studies should consider multi-method assessment of study variables. The current study utilized self-report measures, which may not accurately reflect assertive behavior or realistically assess sex-related fears. Self-report methodology may be a particular concern for assessment of cognitive emotion dysregulation, given that individuals lacking in emotional awareness may not be accurate reporters. However, other aspects of cognitive emotion dysregulation, such as nonacceptance, would likely be accurate. Future studies might address this concern by using psychophysiological or experimental methods to assess emotion dysregulation, and analog methods to assess assertive responding. Perhaps most importantly, findings should be considered preliminary until prospective studies can verify the temporal relationships implied here. Whether fear of sexual powerlessness decreases the likelihood of assertive behavior or vice versa cannot be determined with a cross-sectional design. However, as research has consistently demonstrated a reciprocal relationship between sexual assertiveness and sexual victimization (Greene \& Navarro, 1998; Livingston et al., 2007), the cause or effect may be less relevant.

\section{Implications}

Interventions and risk-reduction programs should highlight the sexual power that is available and due to a young woman, focus on sexually assertive response options, and explore the influence of emotion regulation. Phillips (2000) puts forward the notion that "women need to be positioned in education, legal, scholarly, and popular discourses as co-subjects and active initiators in their own sexualities, rather than simply respondents to men's requests or demands for sex" (p. 200, emphasis in original). Thus, programming should specifically address young women's subjective feelings of sexual vulnerability and explore ways of performing sexual assertiveness that feel accessible within the reality of a sexual situation. One of the most prominent recommendations of the literature on fear appeals is to promote higher perceptions of 
self-efficacy in conjunction with fear appeal exposure (Cho \& Salmon, 2006). A focus on factors that may increase perceptions of sexual power (e.g., sexual assertiveness, risk appraisal, and effective resistance strategies) may strengthen young women's sense of sexual self-efficacy. Indeed, research shows that interventions focusing on sexual assertiveness and self-efficacy can reduce women's risk for sexual victimization (Orchowski, Gidycz, \& Raffle, 2008). Nonetheless, it is important to emphasize that such messages are not meant to suggest victims have done anything wrong, nor that they should have done anything that they did not. Rather, the implication is only that sexual assertiveness has been helpful in such situations.

Messages about sexual assertiveness may need to be tailored to particular audiences. Clearly women with a history of sexual victimization struggle more extensively with fear of sexual powerlessness and cognitive emotion dysregulation. For victims, when these two factors interact, sexual compliance is promoted. This is a great concern, as it contributes to unwanted sexual experiences and given research that demonstrates that lack of sexual assertiveness contributes to risk of revictimization (Greene \& Navarro, 1998; Livingston et al., 2007). In view of the fact that victims are at an increased risk of future sexual victimization, it is particularly important that research identify barriers to their ability to be sexually assertive. These findings highlight the need for targeted intervention for victims.

It is important to design messages about sexuality that stress that individuals involved in sexual interactions have the power to decide both whether they will engage in sexual activity and, if so, what extent of sexual activity they desire. Such an approach is consistent with empowerment-based prevention interventions (Rozee \& Koss, 2001). It would be overly simplistic to suggest that feelings of empowerment in sexual situations will protect young women from sexual violence. Sadly, Phillips' (2000) inquiry into college women's sexual experiences showed that for many women, a sense of power that does not incorporate the cultural context of gender power asymmetry can leave women unprepared for or less aware of potential dangers, and thus paradoxically, steer them into experiences of victimization. Thus, it is essential that we acknowledge the social expectations placed on women to conform to certain gender and sexual roles and their impact on young women's ability to be sexually assertive or noncompliant. By focusing on changeable aspects of sexual victimization, we seek not to blame victims, but rather to direct our work to whatever protective mechanisms may be possible (Frazier, Mortensen, \& Steward, 2005). Given these findings, the most critical thing we can do is focus on the fear of sexual powerlessness that women with and without histories of sexual victimization endorsed. Only messages that they have the right to make choices about when, in what ways, and with whom they share their bodies will empower young women to utilize sexual assertiveness.

\section{Acknowledgments}

The authors would like to acknowledge numerous individuals who assisted with data collection and coding, including Kathleen DeNardi, Dave Walker, Alison Kaiser, Erika Kelley, Emily Prather, Melissa Roemmele, Misti Rogers, Emily Lambrecht, Rachel Chait, Kristi Wolfe, Ashley Newlove, Sarah Griffeth and Kate Rosenblatt. The authors are grateful to Noam 
Zerubavel, Raven Cuellar, Angela Volz, David Waller, and Yael and Eviatar Zerubavel for their thoughtful and insightful feedback on earlier drafts. We appreciate the helpful recommendations made by the master's thesis committee members, Margaret O'Dougherty Wright and Amanda Diekman.

\section{Authors' Note}

This article is substantially based on the first author's master's thesis, written under the supervision of the second author. An earlier version of the article was presented at the Midwestern Psychological Association Conference in 2010.

\section{Declaration of Conflicting Interests}

The author(s) declared no potential conflicts of interest with respect to the research, authorship, and/or publication of this article.

\section{Funding}

The author(s) received no financial support for the research, authorship, and/or publication of this article.

\section{References}

Aldao, A., \& Nolen-Hoeksema, S. (2010). Specificity of cognitive emotion regulation strategies: A transdiagnostic examination. Behaviour Research and Therapy, 48, 974-983.

Austin, S. (2005). Women's aggressive fantasies: A post-Jungian exploration of self-hatred, love and agency. New York: Routledge.

Blackledge, J. T., \& Hayes, S. C. (2001). Emotion regulation in acceptance and commitment therapy. Journal of Clinical Psychology, 57, 243-255.

Burton, C. W., Halpern-Felsher, B., Rankin, S. H., Rehm, R. S., \& Humphreys, J. C. (2011). Relationships and betrayal among young women: Theoretical perspectives on adolescent dating abuse. Journal of Advanced Nursing, 67, 1393-1405.

Cahill, A. J. (2001). Rethinking rape. Ithaca, NY: Cornell University Press.

Cho, H., \& Salmon, C. T. (2006). Fear appeals for individuals in different stages of change: Intended and unintended effects and implications on public health campaigns. Health Communication, 20, 91-99.

Chung, D. (2007). Making meaning of relationships: Young women's experiences and understandings of dating violence. Violence Against Women, 13, 1274-1295.

Cohen, J. (1992). A power primer. Psychological Bulletin, 112, 155-159.

Coleman, P. T. (2006). Power and conflict. In M. Deutsch, P. T. Coleman, \& E. C. Marcus (Eds.), The handbook of conflict resolution: Theory and practice (pp. 120-143). Hoboken, NJ: Wiley.

Cooper, M. L., Shapiro, C. M., \& Powers, A. M. (1998). Motivations for sex and risky sexual behavior among adolescents and young adults: A functional perspective. Journal of Personality and Social Psychology, 75, 1528-1558.

Fisher, B. S., Cullen, F. T., \& Turner, M. G. (2000). The sexual victimization of college women. Washington, DC: U.S. Department of Justice, National Institute of Justice.

Foa, E. B., \& Rothbaum, B. O. (1998). Treating the trauma of rape: Cognitive-behavioral therapy for PTSD. New York: Guilford Press. 
Foa, E. B., Zinbarg, R., \& Rothbaum, B. O. (1992). Uncontrollability and unpredictability in post-traumatic stress disorder: An animal model. Psychological Bulletin, 112, 218-238.

Frazier, P. A., Mortensen, H., \& Steward, J. (2005). Coping strategies as mediators of the relations among perceived control and distress in sexual assault survivors. Journal of Counseling Psychology, 52, 267-278.

Frazier, P. A., Tix, A. P., \& Barron, K. E. (2004). Testing moderator and mediator effects in counseling psychology research. Journal of Counseling Psychology, 51, 115-134.

Garnefski, N., Kraaij, V., \& Spinhoven, P. (2001). Negative life events, cognitive emotion regulation and emotional problems. Personality and Individual Differences, 30, 1311-1327.

Gratz, K. L., \& Roemer, L. (2004). Multidimensional assessment of emotion regulation and dysregulation: Development, factor structure, and initial validation of the Difficulties in Emotion Regulation Scale. Journal of Psychopathology and Behavioral Assessment, 26, 41-54.

Greene, D. M., \& Navarro, R. L. (1998). Situation-specific assertiveness in the epidemiology of sexual victimization among university women. Psychology of Women Quarterly, 22, 589-604.

Herman, J. L. (1992). Trauma and recovery. New York: Basic Books.

Holmbeck, G. N. (1997). Toward terminological, conceptual, and statistical clarity in the study of mediators and moderators: Examples from the child-clinical and pediatric psychology literatures. Journal of Consulting and Clinical Psychology, 65, 599-610.

Impett, E. A., \& Peplau, L. A. (2003). Sexual compliance: Gender, motivational, and relationship perspectives. Journal of Sex Research, 40, 87-100.

Impett, E. A., Schooler, D., \& Tolman, D. L. (2006). To be seen and not heard: Femininity ideology and adolescent girls' sexual health. Archives of Sexual Behavior, 35, 131-144.

Isaacs, K. S. (1998). Uses of emotion: Nature's vital gift. Westport, CT: Praeger.

Koss, M. P., Gidycz, C. A., \& Wisniewski, N. (1987). The scope of rape: Incidence and prevalence of sexual aggression and victimization in a national sample of higher education students. Journal of Consulting and Clinical Psychology, 55, 162-170.

Krebs, C. P., Lindquist, C. H., Warner, T. D., Fisher, B. S., \& Martin, S. L. (2007). Campus sexual assault study, final report. Washington, DC: U.S. Department of Justice.

Kyes, K. B. (1995). Using fear to encourage safer sex: An application of protection motivation theory. Journal of Psychology \& Human Sexuality, 7, 21-37.

Linehan, M. M. (1993). Cognitive-behavioral treatment of borderline personality disorder. New York: Guilford Press.

Livingston, J. A., Testa, M., \& VanZile-Tamsen, C. (2007). The reciprocal relationship between sexual victimization and sexual assertiveness. Violence Against Women, 13, 298-313.

Macy, R. J., Nurius, P. S., \& Norris, J. (2007). Latent profiles among sexual assault survivors: Implications for defensive coping and resistance. Journal of Interpersonal Violence, 22, 543-565.

McClelland, G. H., \& Judd, C. M. (1993). Statistical difficulties of detecting interactions and moderator effects. Psychological Bulletin, 114, 376-390.

Messman-Moore, T. L., Walsh, K. L., \& DiLillo, D. (2010). Emotion dysregulation and risky sexual behavior in revictimization. Child Abuse \& Neglect, 34, 967-976.

Messman-Moore, T. L., Ward, R. M., \& Walker, D.P. (2007, November). Sexual assertiveness in relational context: Development and psychometric properties of the Sexual Assertiveness Questionnaire for Women $(S A Q-W)$. Paper presented at the 50th annual meeting of the Society for the Scientific Study of Sexuality, Indianapolis, IN. 
Muehlenhard, C. L., Powch, I. G., Phelps, J. L., \& Giusti, L. M. (1992). Definitions of rape: Scientific and political implications. Journal of Social Issues, 48, 23-44.

Nurius, P. S. (2000). Risk perception for acquaintance sexual aggression: A social-cognitive perspective. Aggression and Violent Behavior, 5, 63-78.

Orchowski, L. M., Gidycz, C. A., \& Raffle, H. (2008). Evaluation of a sexual assault risk reduction and self-defense program: A prospective analysis of a revised protocol. Psychology of Women Quarterly, 32, 204-218.

O'Sullivan, L. F., \& Byers, E. S. (1996). Gender differences in responses to discrepancies in desired level of sexual intimacy. Journal of Psychology \& Human Sexuality, 8, 49-67.

Phillips, L. M. (2000). Flirting with danger: Young women's reflections on sexuality and domination. New York: New York University Press.

Reinholtz, R. K., Muehlenhard, C. L., Phelps, J. L., \& Satterfield, A. T. (1995). Sexual discourse and sexual intercourse: How the way we communicate affects the way we think about sexual coercion. In P. J. Kalbfleisch \& M. J. Cody (Eds.), Gender, power, and communication in human relationships (pp. 141-162). Hillsdale, NJ: Lawrence Erlbaum.

Rhatigan, D. L., \& Street, A. E. (2005). The impact of intimate partner violence on decisions to leave dating relationships: A test of the investment model. Journal of Interpersonal Violence, 20, 1580-1597.

Rozee, P. D., \& Koss, M. P. (2001). Rape: A century of resistance. Psychology of Women Quarterly, 25, 295-311.

Rudman, L. A., \& Fairchild, K. (2007). The F word: Is feminism incompatible with beauty and romance? Psychology of Women Quarterly, 31, 125-136.

Schmidt, G. (1995). Commentary: Emancipation and social change in heterosexual relationships. Journal of Psychology \& Human Sexuality, 7(3), 7-20.

Stegge, H., \& Terwogt, M. M. (2007). Awareness and regulation of emotion in typical and atypical development. In J. J. Gross (Ed.), Handbook of emotion regulation (pp. 269-286). New York: Guilford Press.

Tice, D. M., \& Bratslavsky, E. (2000). Giving in to feel good: The place of emotion regulation in the context of general self-control. Psychological Inquiry, 11, 149-159.

Tolman, D. L. (2002). Dilemmas of desire: Teenage girls talk about sexuality. Cambridge, MA: Harvard University Press.

Ullman, S. E. (1998). Does offender violence escalate when rape victims fight back? Journal of Interpersonal Violence, 13, 179-192.

Ullman, S. E., Karabatsos, G., \& Koss, M. P. (1999). Alcohol and sexual assault in a national sample of college women. Journal of Interpersonal Violence, 14, 603-625.

Walker, D. P., Messman-Moore, T. L., \& Ward, R. M. (2011). Number of sexual partners and sexual assertiveness predict sexual victimization: Do more partners equal more risk? Violence and Victims, 26, 774-787.

Witte, K., \& Morrison, K. (1995). Using scare tactics to promote safer sex among juvenile detention and high school youth. Journal of Applied Communication Research, 23, 128-142.

Zeanah, P. D., \& Schwarz, J. C. (1996). Reliability and validity of the Sexual Self-esteem Inventory for Women. Assessment, 3, 1-15.

\section{Author Biographies}

Noga Zerubavel, $\mathrm{PhD}$, received her doctorate in clinical psychology from Miami University, and is currently a clinical associate in the Department of Psychiatry and Behavioral Sciences at 
Duke University Medical Center. Her research interests focus on identifying risk factors related to sexual victimization and intimate partner violence. Her clinical work has been focused on childhood sexual abuse, adult sexual victimization, and intimate partner violence. She is interested in sequelae of interpersonal trauma such as emotion dysregulation, dissociation, and interpersonal problems. She is also interested in interpersonal, feminist, and mindfulness-based approaches to intervention.

Terri L. Messman-Moore, $\mathrm{PhD}$, is the O'Toole Family Professor of psychology, the director of clinical training at Miami University, and a clinical psychologist. Her research interests focus on factors that may contribute to women's vulnerability for sexual revictimization following a history of childhood sexual abuse. She is particularly interested in studying emotion dysregulation, interpersonal difficulties, risky sexual behavior, and substance use among female emerging adults with histories of sexual trauma. 